\title{
Asymmetries and errors in perception of depth from disparity suggest a multicomponent model of disparity processing
}

\author{
DAVID D. LANDERS and LAWRENCE K. CORMACK \\ University of Texas, Austin, Texas
}

\begin{abstract}
In three experiments, asymmetries between the processing of crossed and uncrossed disparities were investigated. The target was a luminance-defined circle concentric to a fixation mark, viewed stereoscopically on a computer monitor for $105 \mathrm{msec}$. Fifteen disparities were presented according to the method of constant stimuli. Observers indicated the apparent direction of target depth relative to fixation. All experiments measured both the accuracy and latency of this response. Experiment 1 showed fewer errors and shorter reaction times for identifying crossed disparities. Experiments 2 and 3 replicated Experiment 1 and also showed that observers may often perceive a target in the direction opposite that prescribed by the disparity information. We propose that the asymmetries and reversals result from differences in computation of sign, not of magnitude. This notion is consistent with a scheme of continuous disparity tuning and accounts for such asymmetries and errors without positing disparity pooling mechanisms.
\end{abstract}

A fundamental issue in the study of stereopsis concerns the manner in which retinal disparities are processed by the visual system. The two most popular schemes involve either a small number of relatively independent channels (or pools of disparity detectors), or a large number of distinct yet continuously distributed disparity detectors.

The first scheme was proposed by Richards (1971) on the basis of experiments that revealed anomalous individual differences, or "stereoanomalies," in disparity processing. Richards found that over half of the observers he tested were insensitive to either crossed or uncrossed disparities in local stereograms. In addition, he observed that, for any observer, two very different disparities of the same sign could signal the same depth percept. Some observers even exhibited an apparent reversal of the disparity sign information: The entire range of disparities (crossed and uncrossed) elicited depth in only one direction. Based on these findings, Richards posited at least two distinct and independent pools of integrated disparity detectors (crossed and uncrossed). That is, Richards presumed that, since some observers appeared insensitive to the entire range of disparities of a particular direction, the disparity detectors for that direction were pooled into a single (malfunctioning) mechanism. Moreover, the phe-

This work was supported by NIH/NEI EY 10303 and AFOSR F49620-93-1-0307. We wish to thank S. B. Stevenson for his helpful comments regarding the results and R. P. O'Shea, R. Patterson, and two anonymous referees for their helpful comments regarding an earlier version of the manuscript. Portions of these findings were presented for the Association for Research in Vision and Ophthalmology in Ft. Lauderdale, FL, May 1995. Correspondence should be addressed to D. D. Landers, Department of Psychology and Center for Vision and Image Sciences, Mezes 330, University of Texas, Austin, TX 78712-1189 (e-mail: slanders@mail.utexas.edu). nomenon of the depth metemerism was consistent with the notion of a small number of disparity channels.

Alternatives to Richards's basic theory (or perhaps extensions of it) have been presented by various authors on the basis of considerations from physiology, psychophysics, and modeling. Although early physiological studies adopted Richards's "pooling" terminology to describe single units in visual cortex (Poggio \& Fischer, 1977), it was later found that more precise categories were required (Poggio, Gonzalez, \& Krause, 1988). Moreover, LeVay and Voight (1989) explicitly addressed the pools/ continuum question and concluded that a continuum of disparity tuning existed in the cat visual cortex. Psychophysical studies designed to address this question specifically have found evidence for a continuum (Cormack, Stevenson, \& Schor, 1993; Stevenson, Cormack, Schor, \& Tyler, 1992). Finally, efforts to model psychophysical data using a system of few channels have failed, whereas efforts using systems comprising a larger number of channels have succeeded (Lehky \& Sejnowski, 1990; Stevenson et al., 1992).

Despite the compelling evidence contrary to the pooling hypothesis presented above, many studies have reported asymmetries between crossed and uncrossed disparity processing that are consistent with a system that sharply segregates crossed and uncrossed disparities. For example, Breitmeyer, Julesz, and Kropfl (1975) and Julesz, Breitmeyer, and Kropfl (1976) found that uncrossed disparities in random-dot stereograms (RDSs) elicited lower stimulus duration thresholds in the upper visual field than in the lower visual field, whereas the reverse was true for crossed disparities. Previc, Breitmeyer, and Weinstein (1995) supported the above finding, but only for uncrossed disparities. Perhaps more importantly, they found 
overall accuracy for discriminating crossed targets to be superior to that for uncrossed targets. Manning, Finlay, Neill, and Frost (1987) measured stimulus duration thresholds for crossed and uncrossed disparities at various positions in the visual field. At every position they tested, longer durations were required to detect uncrossed disparities than crossed disparities. These findings were supported and extended by Patterson et al. (1995). Not only did they find that performance was superior for detecting depth for crossed disparities than for uncrossed disparities, but also that performance was higher for discriminating between two depths when both were located in the crossed direction compared to the uncrossed direction. Moreover, they reported that many observers perceived targets in the opposite direction prescribed by the disparity information, and these reversals were more numerous with uncrossed targets.

We attempted to establish whether or not this crossed disparity advantage would also be detected when the stimulus is a luminance-defined "local" target. Evidence from Breitmeyer et al. (1975) and Julesz et al. (1976) suggests that it would not. Both studies reported that the introduction of monocular cues to the target's form improved perception times and eliminated the reported anisotropies. Julesz et al. argued that the RDS provides a more pure measurement of disparity detector activity since it is uncomplicated by monocular cues (i.e., the target is solely defined by disparity). This suggests that the anisotropies in detection are purely binocular phenomena, and that the effects are exclusive in that they may be extinguished by intrusion of monocular stimulation.

There is an advantage to using a "local" stimulus in that it avoids a potential confound from differences in form detection, which may occur in the popular version of RDS. As pointed out by Manning et al. (1987), there may be inherent differences between detecting a shape in front of a background plane versus detecting a plane behind an aperture of some shape. Other research does suggest that disparity interpolation is more efficient for surfaces of crossed disparity than for those of uncrossed disparity (Yang \& Blake, 1995), attesting to the complex nature of such stimuli. A second advantage is that a local target allows a wider range of disparities (and hence, more disparity values) to be examined. Of the studies cited earlier using RDSs to study disparity processing asymmetries, the maximum number of disparities sampled was 4 ; the present study sampled 15 .

In the present study, reaction time (RT) and percent correct were both used as dependent measures. RT is useful as a measure because it is sensitive enough that it continues to decrease as stimulus strength increases, even long after suprathreshold intensity levels are reached (Snodgrass, 1975). Also, by analyzing both RT and the associated percent correct values, one can obtain a more precise measure of the salience of the stimuli than by analyzing the percent correct alone. It could be the case that studies that have failed to report asymmetries in disparity processing have done so because the asymmetry is manifest in RT, which is not measured. That is, accuracy in each disparity direction could be equated, to a degree, by increasing RTs for the disadvantaged direction.

\section{GENERAL METHOD}

\section{Apparatus}

Stimulus presentation and data collection were performed on an Apple Quadra 950 computer using third-party software (Cedrus Superlab 1.67). Stimuli were viewed at approximately $57 \mathrm{~cm}$ through a mirror stereoscope on a Macintosh 13-in. monitor in a dimmed room. The stereoscope was covered with a black fabric hood in order to prevent extraneous light from interfering with stimulus presentation. In addition, apertures and black light baffles were incorporated to reduce distracting reflections within the stereoscope. The mirrors of the stereoscope were adjusted so that convergence was appropriate for a $57.3-\mathrm{cm}$ viewing distance.

\section{Stimuli}

All stimuli were drawn with $4.3^{\prime}$ thick white lines (straight or curved) against a black background, providing $99.7 \%$ Michelson contrast. Two examples, one crossed and one uncrossed, are depicted in Figure 1.

Fixation was defined by a binocular $26.1^{\prime}$ square outline located in the central visual field, flanked by two vertical nonius lines. These were $32.6^{\prime}$ long and began $13.0^{\prime}$ eccentric from the outer edge of the square.

The target consisted of a $93.5^{\prime}$-diameter circle; its average disparity always caused it to appear concentric to the fixation square. That is, with the exception of the smallest disparity used, each half image of the target was displaced half of the total disparity in order to keep the fused image's visual direction equal to that of the fixation mark. Crossed and uncrossed disparities of $2.2^{\prime}$ (only the right half image was displaced to produce this disparity), $4.3^{\prime}, 8^{\prime} .7^{\prime}, 17.4^{\prime}, 34.8^{\prime}$ (diplopic), 52.2' and 69.6' were used, as well as a stimulus with zero disparity. Each half-image in its entirety (target and fixation) had a mean luminance of $0.6 \mathrm{~cd} / \mathrm{m}^{2}$ when averaged over the visible area of the screen. All target durations were $105 \mathrm{msec}$ in order to avoid vergence eye movements during stimulus presentation. (Target durations and recorded response times were calibrated with an oscilloscope and a United Detector Technology photocell, PIN-10.)

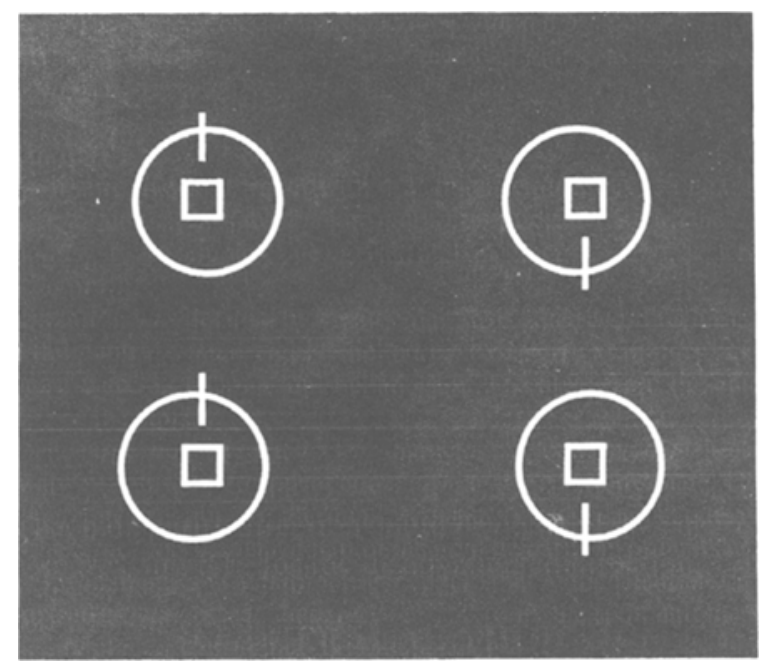

Figure 1. Examples of stimuli. Under crossed fusion, the top stimulus produces an uncrossed disparity relative to fixation (distal target); the bottom stimulus produces a crossed disparity relative to fixation (proximal target) of the same magnitude. When viewed at $57.3 \mathrm{~cm}$, each provides $8.7^{\prime}$ of disparity. 


\section{Data Analysis}

All RTs less than 200 or greater than $2,000 \mathrm{msec}$ were subsequently removed. RTs less than $200 \mathrm{msec}$ were presumed to result from anticipation (Snodgrass, 1975), and those greater than 2,000 msec were presumed to result from inattention or other extraneous factors.

RTs for error responses were not removed from data analysis for three reasons. First, we feel that in order to evaluate fairly the speed-accuracy function, all responses should be included in the RT analysis. Second, when "incorrect" responses are removed, only those responses to ambiguous stimuli that are incorrect by chance are removed. Consequently, there are still responses (in the "correct" category) that represent unperceived stimuli to which the observer guessed correctly. Third, there is no reliable way to identify errors that occur because the observer perceives the signal in a manner that genuinely contradicts the percept prescribed by the stimulus. Therefore, analyzing all response RTs is probably the most conservative method for interpreting data in a forced-choice, RT experiment such as in the present study.

To investigate the effect of this analysis, two observers' data (D.D.L. and C.R.H.) were analyzed without the incorrect responses; the resulting curves were virtually identical to the originals.

\section{EXPERIMENT 1}

Experiment 1 was designed to detect differences in identifying crossed and uncrossed disparities, using both RT and percent correct as dependent measures.

\section{Method}

Observers. Three naive observers (paid volunteers) and 2 informed observers (the authors) participated. All observers had normal or corrected-to-normal acuity (at least 20/20 in both eyes) and could perceive depth in anaglyphic stereograms (from Julesz, 1971).

Procedure. The 15 disparities and one additional zero-disparity stimulus composed a block, presented according to the method of constant stimuli. Ten blocks composed a single run, for a total of 160 trials. A rest break followed the fifth block. Observers typically completed three to five runs per day.

On each trial, observers responded "front" or "back" via a keypress to indicate the direction of depth of the target circle relative to the fixation square. In order to control for spatial stimulus-response compatibility effects, observers were instructed to hold the keypad so that the responses were in spatial correspondence to the stimuli (see Heister, Ehrenstein, \& Schroeder-Heister, 1986). ${ }^{1}$ They were instructed to respond as quickly as possible while maintaining a minimal error rate. Observers were aware that RT and accuracy were being recorded.

At the beginning of the run, only the fixation mark (including the nonius lines) was visible. It remained on the screen until the observer achieved proper fixation and was ready to begin. Upon an initial keypress, $2.025 \mathrm{sec}$ elapsed before the first target appeared. The subsequent targets did not appear until $2.025 \mathrm{sec}$ after the observer responded to the current trial. This intertrial interval was held constant, on the assumption that the observers' need to make a decision in order to respond would prevent invalid anticipatory responses. The fixation mark was visible throughout the entire run, except during the response phase of the trial, in which the screen was completely black.

Before they began the experimental runs, the naive observers participated in a shortened training run that allowed them to become comfortable with the apparatus and stimuli. This run consisted of 12 blocks of 8 disparities each $\left(0^{\prime}, 4.3^{\prime}, 8.7^{\prime}\right.$, and $17.4^{\prime}$ disparities, crossed and uncrossed). The only feedback they received was that they were told they performed "very well." Observers were instructed to cease responding and to notify the experimenter if proper fusion could not be maintained. All observers participated in at least 15 experimental runs.

\section{Results}

The data for Experiment 1 are shown in Figure 2, which plots RT against disparity for the experienced (Figure 2A) and naive (Figure $2 \mathrm{~B}$ ) observers. The insets plot percent correct against disparity for each observer (the zerodisparity conditions are not plotted, as no correct response was possible). For all observers shown, there is a distinct RT advantage for responding to crossed stimuli. In addition, for all observers except L.K.C. (whose errors are minimal and relatively symmetric), factoring in the errors by incorporating a speed-accuracy tradeoff correction would accentuate this crossed advantage.

For all observers, performance generally improves as disparity increases in each direction, yet at a greater rate and magnitude for crossed disparities. For all observers except DDL, performance deteriorates again as stimuli become increasingly diplopic in the uncrossed direction.

Two observers, D.D.L. and C.R.H., did exhibit a bias to respond "front" to the zero-disparity stimulus. However, it is clear from the data of the remaining $2 \mathrm{ob}-$ servers (who exhibited no such bias) that a bias is not responsible for the effect. Likewise, fixation disparity could not explain the results either, since the disparity values over which the effect is manifest are much greater than those disparity values that would be expected to be affected by a fixation disparity.

One naive observer's data are not shown; they are virtually symmetric and without systematic variance in both RT and errors (except for the expected decrease in performance at the smallest disparities). Interestingly, though, the data for this observer's initial six runs are similar to the other naive observers' complete data sets, yet the effect is extinguished by the final nine runs.

During the collection of pilot data for Experiment 1, many naive observers reported that the proximal stimuli were more easily perceived than distal stimuli. In fact, some could not even perform the task because most, if not all, of the targets appeared either proximal or without depth. This suggested an insensitivity, or even a perceptual reversal, of the uncrossed disparity information. The objectives of Experiment 2 were to explore further the asymmetry measured in Experiment 1 and to investigate whether observers may perceive some magnitude of depth from disparity while reversing its sign.

\section{EXPERIMENT 2}

\section{Method}

Observers. Twenty-nine naive observers participated in partial fulfillment of a course credit. They were screened for global stereopsis, as in Experiment 1, yet more inferior visual acuity was accepted (better than 20/32 in each eye). Eight of the original 29 observers were rejected because of poor acuity $(n=5)$, inability to fixate properly $(n=2)$, or inability to perceive depth (one observer was screened as "stereoblind").

Procedure. The procedure was identical to that of Experiment 1 , except that the extra zero-disparity trial was removed to shorten the run, resulting in a total of 150 trials.

In addition, measures were taken to ensure that observers would respond to stimuli only as they truly perceived them, allowing the incidence of perceptual reversals to be measured. In order to stress 

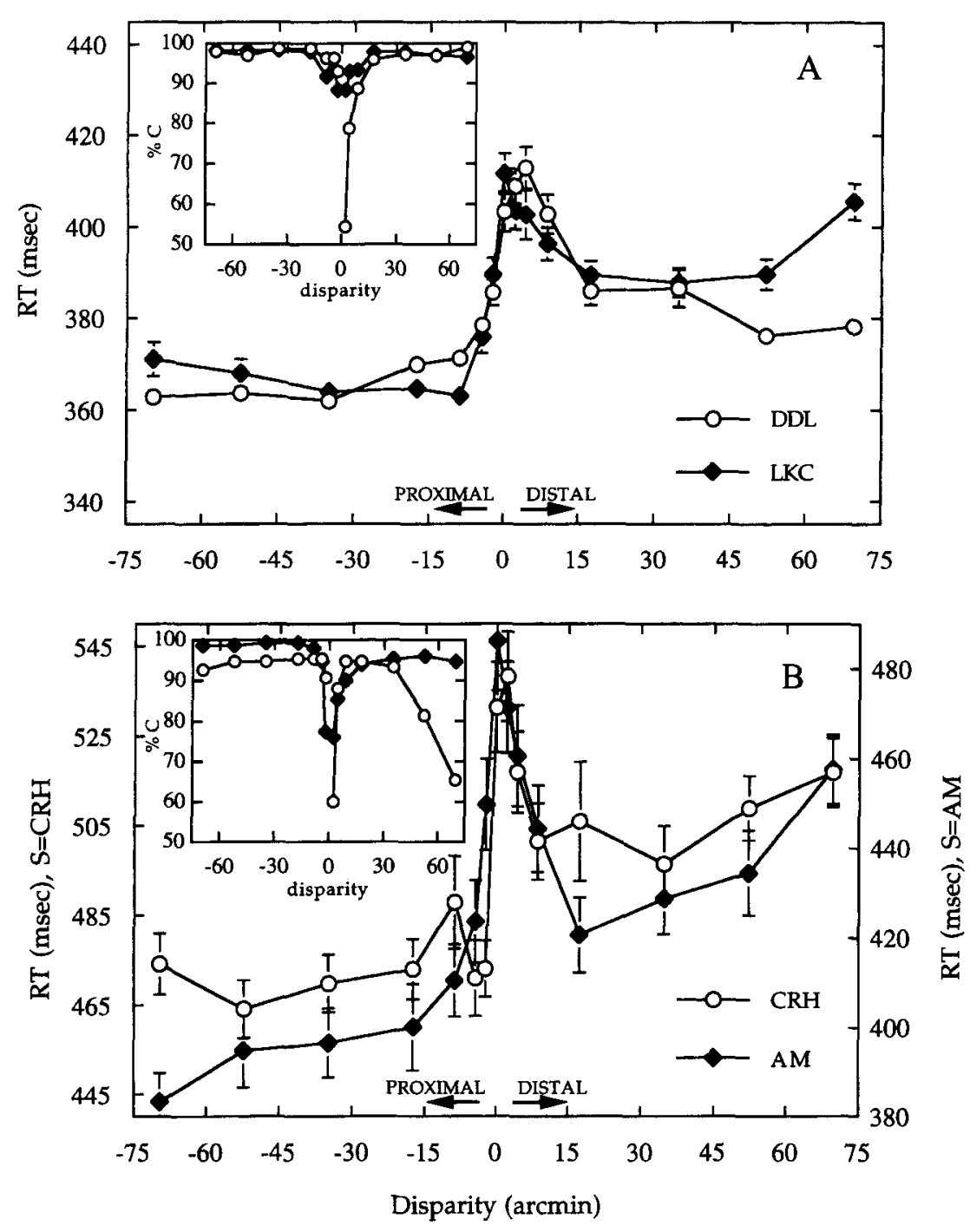

Figure 2. Reaction time (RT) versus disparity for 4 observers. In this and all subsequent figures, negative abscissa values represent crossed disparities. Inset graphs plot percentage correct, using the same abscissae and symbols as the main graphs. Error bars $( \pm 1 S E)$ that are not visible are smaller than the plot symbols. (A) Informed observers. Each point represents the average of 300 trials per disparity for D.D.L. and 260 trials per disparity for L.K.C. (B) Naive observers. Each point represents the average of 150 trials per disparity for each observer. Note that the data of these observers have been shifted, but not scaled, relative to one another; the left ordinate applies to C.R.H., the right to A.M.

accuracy, observers were told that only errors, not RTs, were being measured and that they should proceed slowly enough to avoid mistakes. In order to reduce guessing between the "front" and "back" responses, they were told (incorrectly) that the proportion of proximal to distal targets was randomized on each run so that they may be presented with long sequences or generally biased samples of same-direction targets. In addition, observers were instructed to press a third key with the preferred finger of their left hand to respond "unsure" in any of the "likely" events that there was no depth to judge, or in the event that they were forced to guess. They were instructed to respond with the "front" or "back" keys only when they were reasonably confident of that judgment.

In an attempt to increase observer motivation and to emphasize accuracy further, they were told (incorrectly) that they could leave early $(\approx 20 \mathrm{~min})$, yet still receive full credit, if their accuracy was "sufficient" after three complete runs. After each of the first two runs, the experimenter evaluated the responses. However, each observer always received the same feedback regardless of performance, which was simply that they were performing "OK." Following the second run, they were informed that if their performance on the third run was at least as good as that on the second, they would be finished. In actuality, three runs were collected from each observer, regardless of performance. Data were collected from each observer during one session in usually under $75 \mathrm{~min}$. All observers were informed of the deceptions and the reasons therefor at a debriefing following the experiments.

Training consisted of fixation training and one practice block of stimulus presentations. Observers were informed of how the fixation mark should appear and were coached on how to correct any problems with vergence. The experiment proceeded when the 
observer was able to achieve proper, stable fixation, based on his/her verbal report. Next, observers viewed and responded to one block of stimulus presentations. This block consisted of one of each stimulus used in the experiment ( 15 trials), presented in a pseudorandom order that was identical for each observer. The experimenter sat with the observers while they practiced, but did not provide feedback. When the practice block was over, the experimenter dimmed the light and left the room, allowing the observer to begin the experimental trials. As in Experiment 1, observers were instructed to cease responding and to notify the experimenter if they became unable to maintain proper fixation; such observers' data were subsequently excluded from data analysis.

\section{Results}

Three distinct classes of observer emerged based on the frequency of responses (front, back, or unsure) as a function of disparity. Observers were classified according to the disparity direction to which they assigned the most correct responses, averaged across the range of disparities for each direction.

Group 1 included those observers (15/21) who each exhibited an advantage for correctly discriminating crossed targets. All but 2 also responded "front" to uncrossed targets more often than they responded "back" to crossed targets (which we will later argue are perceptual reversals rather than biased responses to ambiguous stimuli). Their data, collapsed across the 15 observers, are shown in Figure 3.

As in Experiment 1, for the crossed disparities, performance quickly improved with increasing disparity and reached a plateau near $75 \%$ correct. For the uncrossed disparities, performance rose slowly to a peak with increasing disparity, then declined. Consequently, performance for the crossed disparities was substantially greater than that for the uncrossed disparities in both range and magnitude.

Group 2 included those observers (5/21) who each exhibited an advantage for correctly discriminating uncrossed targets. All but 1 also responded "back" to crossed targets more often than they responded "front" to uncrossed targets. Their data, collapsed across the $5 \mathrm{ob}-$ servers, are shown in Figure 4. Again, performance quickly plateaued with increasing disparity in the crossed direction. In the uncrossed direction, performance rose more slowly to a peak with increasing disparity, then declined. Interestingly, the size of the overall asymmetry in Group 2 appears to have been less than that for Group 1.

Group 3 included the observer $(1 / 21)$ who was unable to perform the task. This observer distributed responses fairly evenly across the entire range of disparities.

A potentially troublesome aspect of the data that demands attention is the tendency for Group 1 observers to respond "front" instead of "unsure" to the zero disparity stimulus (no signal), which may render the reported high incidence of reversals suspect. Yet, this tendency may be perceptually founded (perhaps related to size constancy) or it may be a trivial response bias (e.g., a preexisting preference to use the index finger when unsure) in naive observers. It could also be argued that the bias is not the driving force in the data, but rather that it resulted from the observers' experience of the stimuli. That is, instead of a strong "front" response bias influencing Group $1 \mathrm{ob}-$ servers to respond as such over the entire range of disparities, it could be that they formed the bias because most of the stimuli appeared proximal. This notion is supported by the fact that 12 of the 21 total observers reported (often spontaneously) that proximal targets were more

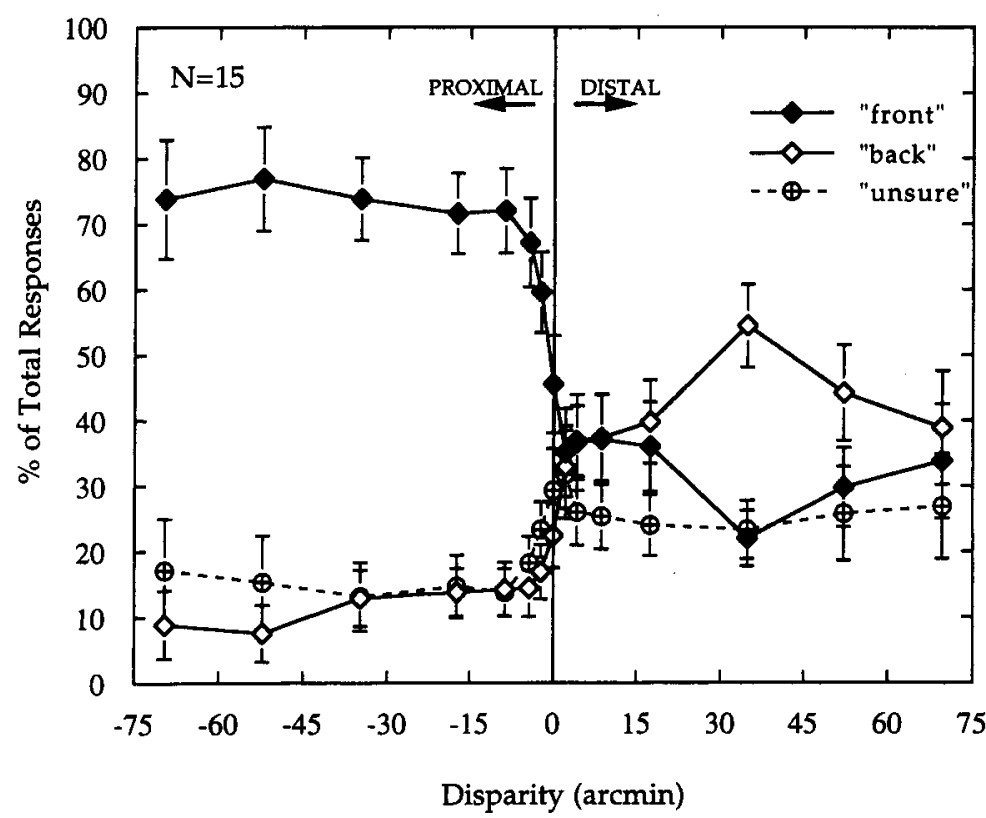

Figure 3. Frequency of responses as a function of disparity for Experiment 2, Group 1 (15 naive observers). Each observer responded to 30 trials at each disparity, providing 450 total trials per disparity. 


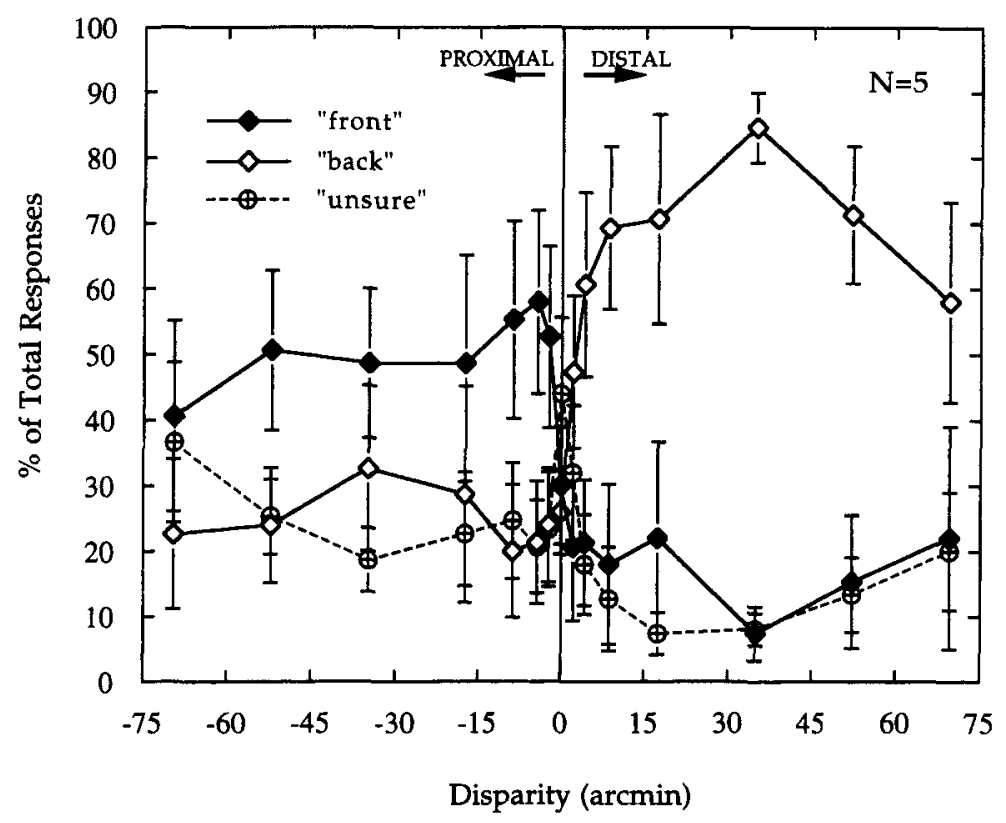

Figure 4. Frequency of responses as a function of disparity for Experiment 2, Group 2 (5 naive observers). Each observer responded to 30 trials at each disparity, providing 150 total trials per disparity.

salient and/or that the majority of the targets appeared proximal, even though their quantities were equal. Two observers reported analogously in favor of distal targets. Additional support comes from the fact that Group 2 observers, who exhibited less of an asymmetry but still showed an effect, did not exhibit a bias. It should also be noted that 6 observers (4 from Group 1 and 2 from Group 2) exhibited an apparent response bias in the opposite direction from their advantaged direction. Nevertheless, regardless of its origin, the presence of a bias is somewhat problematic. For this reason, we attempted to eliminate it experimentally in Experiment 3.

First, however, we turn to the RT data from Experiment 2 for comparison to data from Experiment 1. Figure 5 plots RT (main graphs) and accuracy (inset graphs) against disparity, collapsed across all 15 Group 1 observers (Figure 5A) and across all 5 Group 2 observers (Figure 5B). Only responses made with the index and middle finger of the right hand ("front" and "back") were included in the RT analysis; "unsure" responses (left hand) were omitted (except in the accuracy graphs at zero disparity, where this was the correct response). This was done to avoid complications related to bimanual responding and, hence, to maximize similarity with Experiment 1 . As in Experiment 1, all RTs less than $200 \mathrm{msec}$ were excluded. Because of the greater variability of the data, the maximum acceptable RT $(3,658 \mathrm{msec})$ was set at 3 standard deviations above the mean. Observers were not aware that RT was being measured; accuracy was emphasized over speed.

For Group 1, there is a marked asymmetry in RT corresponding to the asymmetry in accuracy that defines this group of observers. Thus, factoring in the RTs by in- corporating a speed-accuracy tradeoff correction would accentuate the crossed advantage in accuracy seen in Figure 3 (and replotted in the inset of Figure 5A).

For Group 2, there is also a marked asymmetry in RT that accentuates the asymmetry in accuracy. As in the previous RT plots, RTs for the uncrossed disparities decrease with increasing disparity to a minimum, then increase. Yet, for the crossed disparities, RTs increase monotonically with increasing disparity.

As noted, we realized that many observers had not been responding "unsure" as often as they should have been based on the distribution of responses recorded for the zero-disparity stimuli. Although this may have been due to perceptual influences to some degree, it was suspected, on the basis of some observers' reports, that observers may have felt compelled to guess "front" or "back" because of the level of difficulty experienced during the training session. As mentioned earlier, this guessing was often biased in the direction in which the observers most easily detected targets. Experiment 3 addressed the same objective as Experiment 2 but attempted to reduce the amount of guessing between the "front" and "back" responses. This would reduce the influence of response bias on the data and more effectively test whether observers are truly experiencing a perceptual reversal of disparity sign information.

\section{EXPERIMENT 3}

\section{Method}

Observers. Thirty-three naive observers participated in partial fulfillment of a course credit. They were screened in exactly the same manner as those in Experiment 2, except each was also screened for 

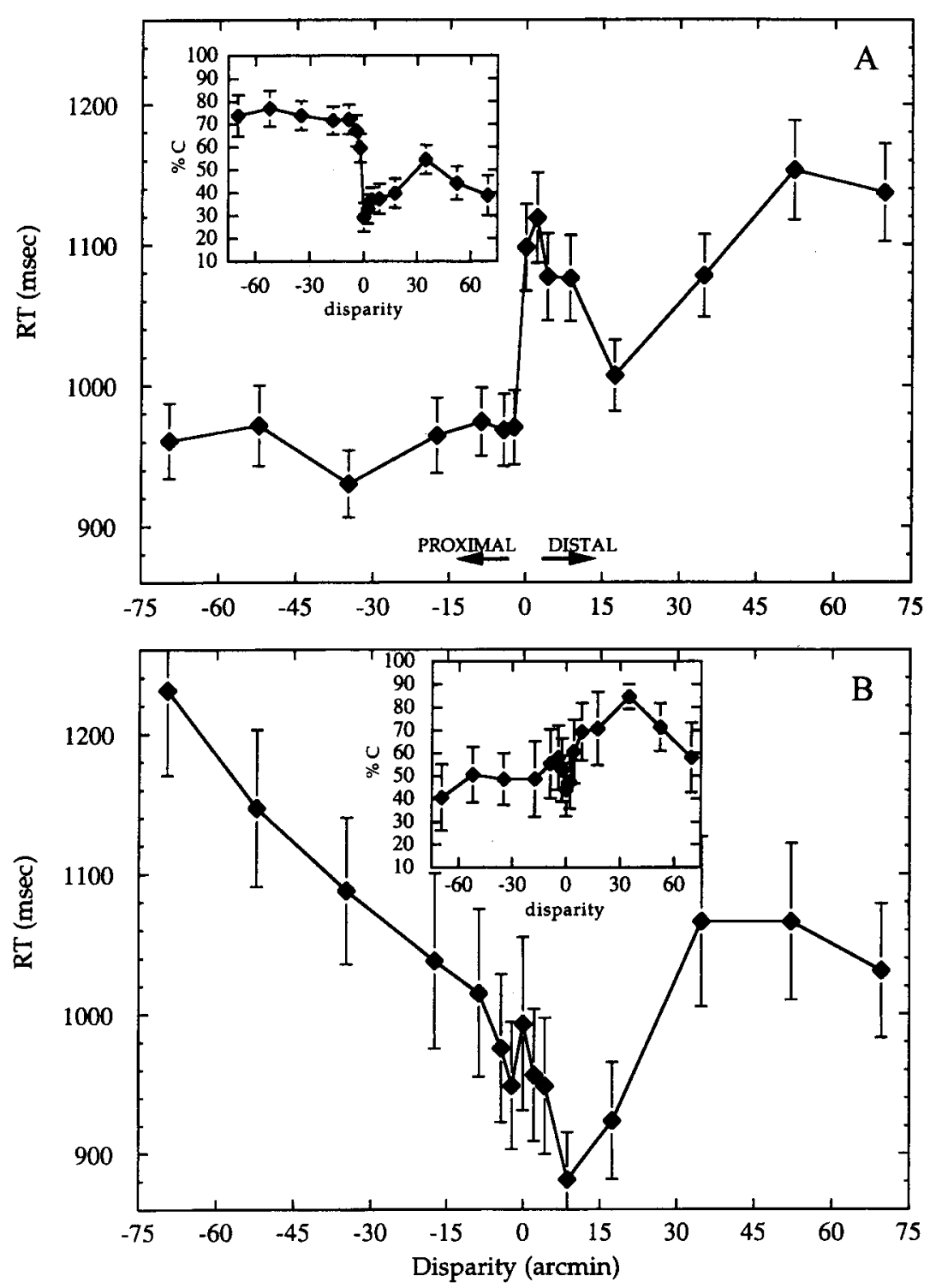

Figure 5. Reaction time (RT) versus disparity for 20 naive observers, Experiment 2. "Unsure" responses were omitted, except at zero disparity in the accuracy graphs (insets). Each point represents, on average, 23 trials per disparity per observer. (A) Group 1 (crossed advantage). (B) Group 2 (uncrossed advantage).

local stereopsis using a test we designed (this test resembled the Circles test by Stereo Optical, Inc.; the smallest disparity tested was $2.2^{\prime}$ ). In addition, if a particular observer had an especially difficult time fusing the fixation mark, a mirror pair of the stereoscope was symmetrically adjusted so that the observer could more easily achieve fusion. Three of the original 33 observers were rejected because of poor acuity $(n=2)$ or inability to maintain fixation $(n=1)$; all observers performed well on the local stereopsis test.

Procedure. The procedure was identical to that of Experiment 2, except more rigorous steps were taken to reduce the incidence of guessing between the "front" and "back" responses. Observers were told (incorrectly) that they would be able to leave early $(\approx 20 \mathrm{~min})$ if, after their third run, they had scored an undisclosed number of points, based on the following scheme. Any response of "unsure" was neutral and resulted in no change in points. Any response of "front" or "back" that was correct resulted in +1 point. Any response of "front" or "back" that was incorrect resulted in -2 points.
It was explained to observers that, with this point system, it was to their advantage to respond conservatively; that is, to respond "front" or "back" only when they were very confident of the target's location. It was also explained that one did not have to be "great" at the task in order to score "well."

\section{Results}

Data collected for Experiment 3 were analyzed and separated in the same way as those in Experiment 2. The success of the counterfeit scoring system in reducing guessing was immediately apparent. In Experiment 2, across all Group 1 and 2 observers, the zero-disparity stimulus was designated "unsure" $33 \%$ of the time, whereas in Experiment 3, it was designated as such $70 \%$ of the time. 
Group 1 (crossed advantage) included 25 of the 30 observers. Their data, collapsed across all 25 observers, are shown in Figure 6. Again, performance increased with increasing disparity in both directions, but more quickly and to a greater magnitude in the crossed direction.

Compared with Group 1 in Experiment 2, performance decreased for the fine disparities in both directions. This indicates, as expected, that the fine disparities are more difficult to detect than the coarse ones for naive observers. Interestingly, at the coarse disparities (i.e., $>34.8^{\prime}$ ), performance was virtually identical for Group 1 observers in both experiments.

Reversed responses decreased in both disparity directions, particularly at the fine disparities. Nevertheless, many such responses to uncrossed targets still did occur under the stricter criterion. This indicates that the depth reversal is a true perceptual phenomenon and is most likely to occur with uncrossed disparities. It is important to note that, as in Experiment 2, many observers (18 of 30 ) reported (often spontaneously) that proximal targets were more salient and/or that the majority of the targets appeared proximal, even though their quantities were equal; only 2 of these observers reported analogously in favor of distal targets.

One might suspect that observers were using cues other than depth to respond to the diplopic stimuli, and that these cues somehow indicated proximal depth. Certainly, other cues could be used, such as degree of diplopia or eccentricity of a target in a half image, but depth would have to be assigned arbitrarily if such a method was used. Also, it is important to note that the $34.8^{\prime}$ stimulus was evidently the most salient of the uncrossed stimuli, yet it was also somewhat diplopic. Therefore, there appears to be nothing intrinsic about diplopia or half-image eccentricity that cues proximal depth. ${ }^{2}$

Group 2 (uncrossed advantage) included only 3 of the 30 Experiment 3 observers. Their data, collapsed across the 3 observers, are shown in Figure 7. Compared with all other groups, they performed poorly. In fact, none of the observers in this group appeared to perceive stimuli correctly over a wide range of disparities. Nevertheless, they were too systematic to be classified as unable to perform the task and were, therefore, included in the analysis. Only 1 of the Group 2 observers exhibited a clear advantage for the uncrossed disparities. The remaining 2 barely met the criterion for classification; their performances were roughly symmetric with regard to the disparity direction domains.

Despite the relatively poor level of performance these data represent, they are consistent in several ways with the other data. Again, it is the finer disparities that seem to be less salient in both disparity directions. Also, the uncrossed performance peaks at the $34.8^{\prime}$ disparity, with some decrease in performance with increasing diplopia. Yet, the crossed performance continued to increase with increasing diplopia. As in Experiment 2, Group 2 exhibited less of an effect than Group 1.

Group 3 (unable to perform the task) included 2 of the 30 Experiment 3 observers. As in Experiment 2, these 2 observers did not present enough systematic variation in their data to indicate that they could discriminate any of the stimuli reliably.

Figure 8 plots RT (main graphs) and accuracy (inset graphs) against disparity, collapsed across all 25 Group 1 observers (Figure 8A) and across all 3 Group 2 observers (Figure 8B). Data were analyzed as in Experiment 2, ex-

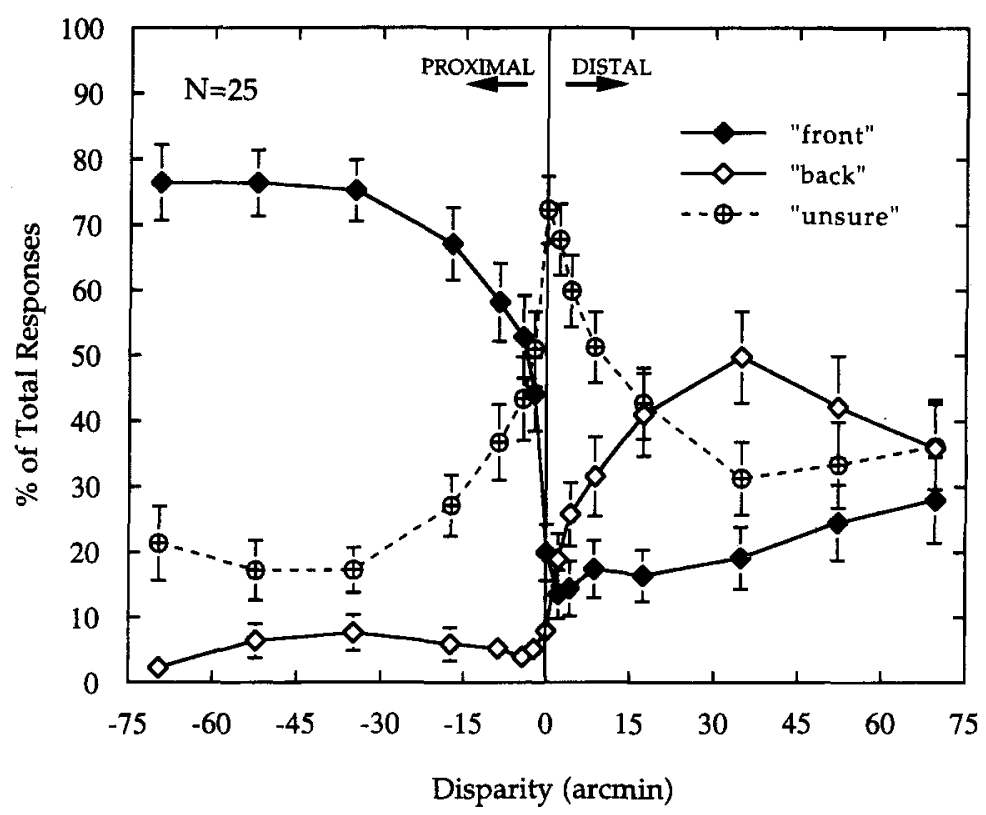

Figure 6. Frequency of responses as a function of disparity for Experiment 3, Group 1 (25 naive observers). Each observer responded to 30 trials at each disparity, providing 750 total trials per disparity. 


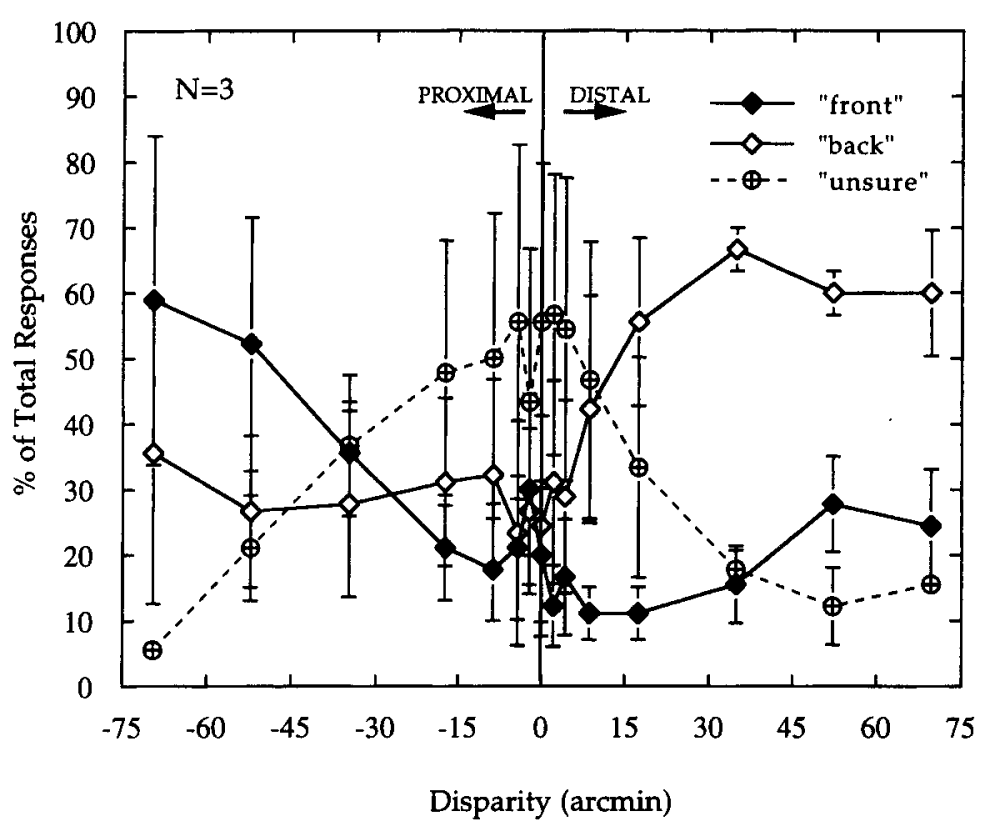

Figure 7. Frequency of responses as a function of disparity for Experiment 3, Group 2 (3 naive observers). Each observer responded to 30 trials at each disparity, providing 90 total trials per disparity.

cept that the maximum acceptable RT (again, 3 standard deviations above the mean) was $5,732 \mathrm{msec}$.

For Group 1, there was a marked asymmetry in RT corresponding to the asymmetry in accuracy that defines this group of observers. Again, factoring in the RTs by incorporating a speed-accuracy tradeoff correction would accentuate the crossed advantage in accuracy.

For Group 2, there was no obvious systematic variation in RT with disparity (the data are presented for completeness). This is probably because of the small number of observers these data represent $(n=3)$. In addition, 1 of the 3 observers responded "unsure" to several stimuli fairly consistently, significantly reducing the number of available data points. Nevertheless, as can be seen in the accuracy graph (inset) of Figure 8, the observers as a group do favor uncrossed disparities.

Figure 9 shows the data from a subset of all observers who exhibited the least amount of bias in responding to the absence of a signal, evident in their ability to correctly identify the zero-disparity stimulus $(n=15)$. Each responded "unsure" to the zero-disparity stimulus at least $80 \%$ of the time. Thirteen of the observers participated in Experiment 3 and 2 in Experiment 2. These data show that the asymmetry and the depth reversal were sustained in the absence of any obvious response bias. Particularly, as disparity increased in the uncrossed direction, observers as a group became more confident in responding to the apparent depth of the target, evident in the decreasing incidence of "unsure" responses. Yet, these confident judgments became increasingly divided between the correct and reversed judgments with increasing disparity.

\section{DISCUSSION}

We have shown that the crossed advantage in disparity processing of global targets in RDSs occurs with luminance-defined local targets as well. In the present study, this phenomenon was evident in both experienced/ informed and inexperienced/naive observers, within and across most observers. The phenomenon is defined by superior performance for responding to crossed disparities compared to uncrossed ones, in RT and accuracy simultaneously. In addition, the disparity range over which crossed stimuli are correctly perceived is broader. Few observers exhibit an analogous advantage in the uncrossed direction; for those who do, the effect is relatively weak. The results also suggest that many observers exhibit some degree of "anomaly" in disparity processing. Although observers may often confuse the sign of a disparity signal of either direction, it is more common to perceive, with some degree of confidence, an uncrossed disparity signal as near than a crossed signal as far.

The asymmetry is seemingly consistent with a scheme of disparity segregation, or pooling, similar to that proposed by Richards (1971), in that it suggests a system in which disparity detectors of the same sign are managed collectively, while each sign as a whole is managed independently. However, we feel that the research cited in favor of a scheme of continuous, tuned disparity detectors without pooling is overwhelming, as there are strong results from physiology (e.g., LeVay \& Voight, 1989), psychophysics (e.g., Cormack et al., 1993), and modeling (e.g., Lehky \& Sejnowski, 1990). Therefore, it may be useful to consider alternative explanations that could 

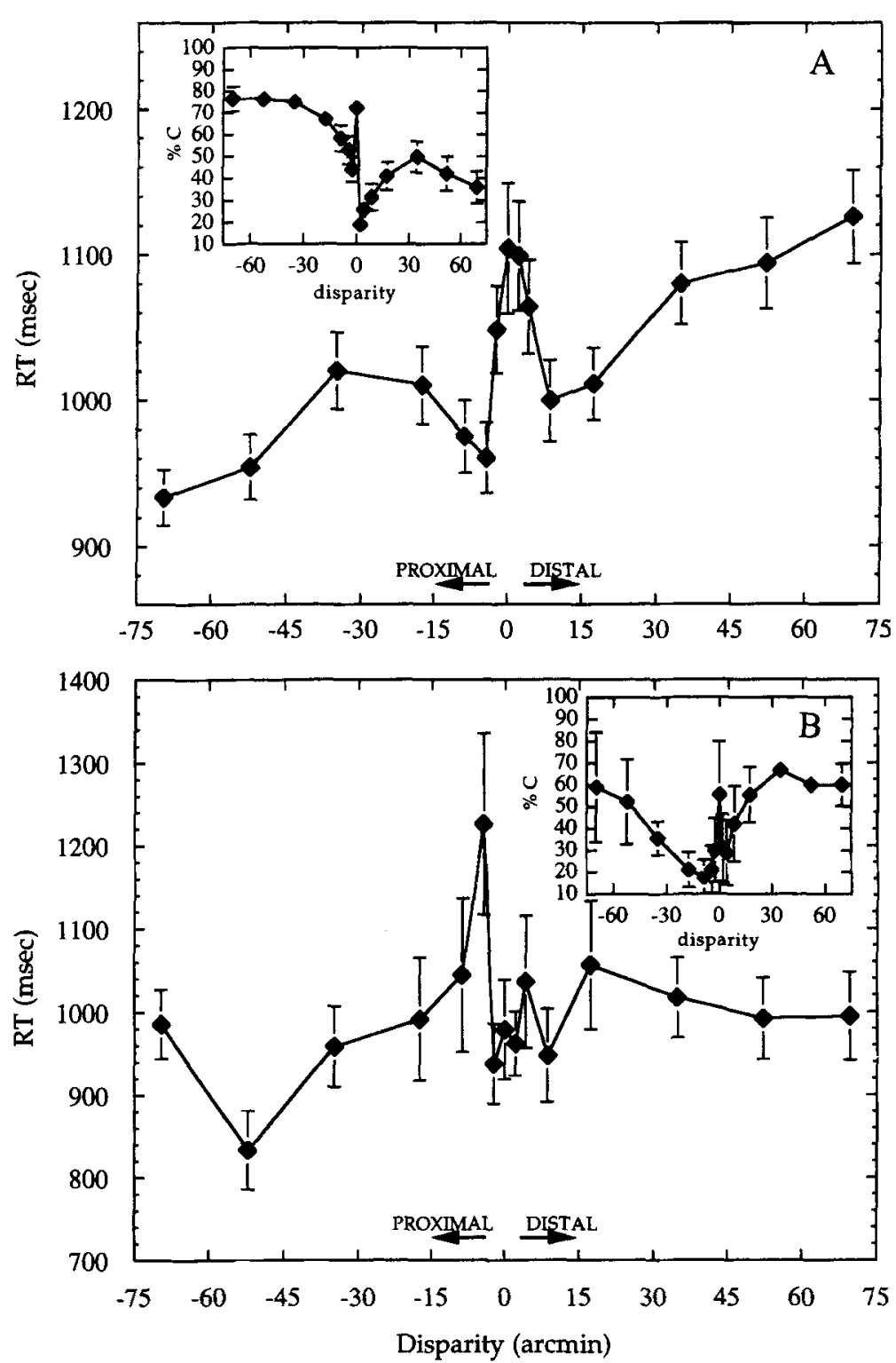

Figure 8. Reaction time (RT) versus disparity for 28 naive observers, Experiment 3. "Unsure" responses were omitted, except at zero disparity in the accuracy graphs (insets). Each point represents, on average, 17 trials per disparity per observer. (A) Group 1 (crossed advantage). (B) Group 2 (uncrossed advantage).

account for the asymmetry without requiring pooling mechanisms.

For example, it is conceivable that the asymmetries and reversals are not disparity-dependent phenomena but result from higher order processes, such as attention. The literature describing attention dynamics in 3-D space is equivocal, however, indicating that the issue is unsettled at present and worthy of independent study. ${ }^{3}$

An alternative theory that also allows continuous tuning and the asymmetry we report to coexist is a theory in which disparity computation is a multicomponent process. In this process, a stage of disparity magnitude computation could be managed by a system of continuous, tuned disparity detectors that respond in a symmetric manner to equidistant targets about the horopter, yielding an unsigned, scalar value. But, in addition, an independent step of disparity sign computation could be managed by a separate mechanism that operates asymmetrically about the horopter (perhaps because of an evolutionary advantage for processing near objects).

Evidence from Patterson et al. (1995) suggests that depth perception is a multicomponent process. They found that duration detection thresholds were lower for merely detecting the presence of a disparity in RDSs (perhaps by detecting a decorrelation at zero disparity) as opposed to perceiving depth from that disparity. Therefore, dis- 


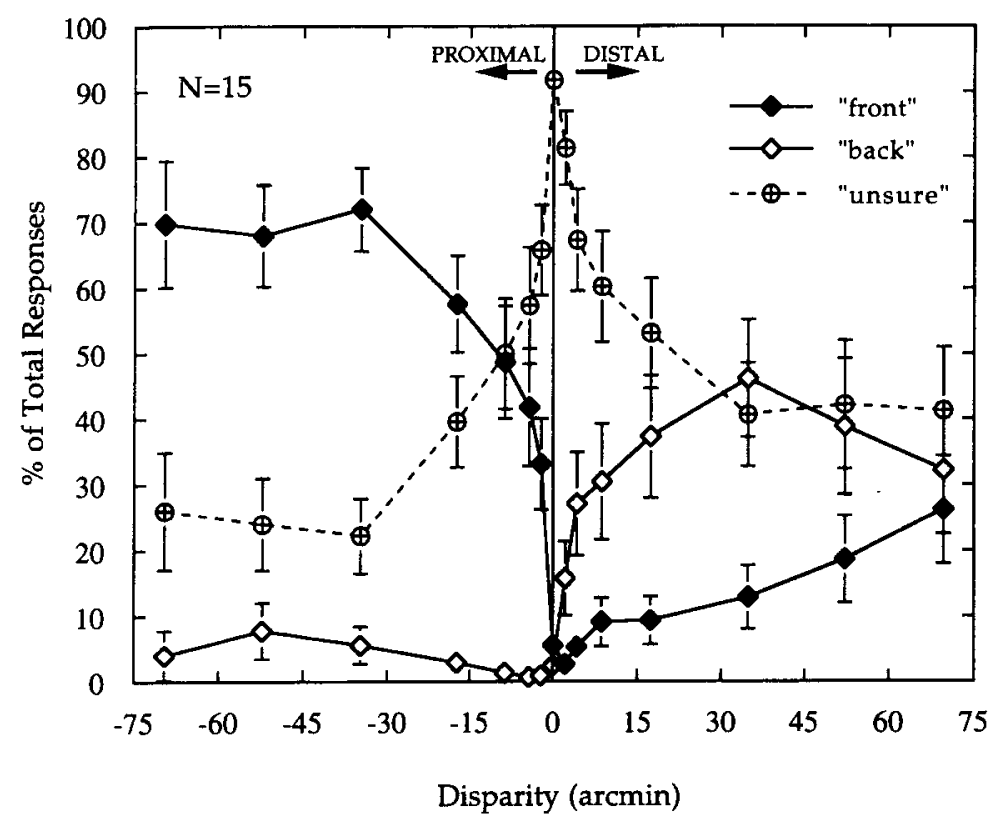

Figure 9. Frequency of responses as a function of disparity for 15 naive observers who correctly identified zero disparity stimuli $\geq 80 \%$ of the time. Each observer responded to 30 trials at each disparity, providing 450 total trials per disparity.

parity detection may be the first step in depth perception, followed by the computation of the magnitude of that disparity. We propose that disparity sign computation, perhaps related to depth mapping, could be a third component in the process. Evidence for this third independent step comes from the documentation of the depth reversal reported in Richards (1971), Patterson et al. (1995), and the present study. That is, fairly often, naive observers perceive a salient magnitude of depth but miscompute its sign, suggesting that the error is distinct from a magnitude computation. In fact, the behavior of "anomalous" observers described originally in Richards (1970) and subsequently in Richards (1971), which served as the foundation upon which disparity pooling was posited, may be described as the result of common disparity sign miscalculations as opposed to failing or "anomalous" neural mechanisms.

Our results and those of others show that "stereoanomaly" is not very unusual, and hence, should not be considered the result of failing neural mechanisms, but rather, normal variability. Even in Richards's (1971) ground-breaking paper, he claimed that "a very large percentage" of observers exhibit this behavior (p. 410); in fact, over half of his observers received the perhaps poorly termed designation. Other research has directly addressed the issue of prevalence. Newhouse and Uttal (1982) and Patterson and Fox (1984) both showed that true stereoblindness is actually quite rare in the population, suggesting that brief stimulus durations, not neural deficiencies, may be responsible for eliciting what appears to be stereoanomalies. Furthermore, Patterson et al. (1995) observed that several of the observers who reversed the sign of depth information were able to perceive depth cor- rectly when the stimulus duration was increased (but still held relatively brief), suggesting that there may be, in certain instances, separate thresholds for perceiving depth and perceiving depth correctly (Patterson, personal communication, November 1995). This observation is important because it further validates the reversal as a true perceptual phenomenon and the idea that "anomaly" may depend on stimulus duration.

Our data support the findings of Newhouse and Uttal (1982) and Patterson and Fox (1984). Of all 62 observers we screened, only 1 exhibited signs of true stereoblindness (and this observer had had corrective surgery for strabismus as a child). In addition, several observers, including one of the authors (D.D.L.), exhibited signs of "stereoanomaly" at one point that disappeared with practice. ${ }^{4}$ Foley and Richards (1974) documented improvement in stereoscopic discrimination with practice; in their study, an observer who was originally diagnosed as stereoblind later developed the ability to see at least some targets in depth after viewing hundreds of such stimuli.

We suggest that the sign confusions occur because observers, particularly naive ones, are unaccustomed to making depth judgments when retinal disparity is the only depth cue. The real-world environment is rich in cues that indicate the sign (direction) of depth. The experimental environment may not provide such cues; inexperienced observers may need to learn to interpret disparity sign information in these settings.

A second discrepancy that needs to be addressed is that studies using near-threshold, local targets do not typically report asymmetries in disparity processing. It may be that the asymmetry is not a phenomenon manifest in the small disparities used to measure stereoacuity. On 
the other hand, it may be common to all stereopsis but is undetected in typical stereoacuity experiments. In many such studies, the data are analyzed in such a way that any asymmetry would be concealed; for example, by fitting a symmetric function to thresholds measured for stereoacuities on both sides of the horopter (e.g., McKee, Welch, Taylor, \& Bowne, 1990). Other studies have analyzed the data in a manner that would be expected to expose any asymmetry, yet simply have not detected one (e.g., Badcock \& Schor, 1985). It should also be noted that other studies have reported asymmetries, but they have been too few and inconsistent across observers to warrant particular analyses; therefore, the data have been averaged across disparity sign (e.g., Legge \& Gu, 1989).

Of particular interest is the situation in which the data simply do not reveal an asymmetry when analyzed in a manner that would be expected to do so. We propose that the asymmetry may be a general phenomenon in stereopsis that goes undetected because percent correct alone as a dependent measure may not completely describe observers' performance. That is, it could be that in experiments where accuracy is stressed, observers take longer to respond to uncrossed stimuli than to crossed stimuli, thereby equating accuracy in such a way that the asymmetry is manifest in RT alone. Typically, this variable is never measured.

In conclusion, most observers apparently exhibit superiority in crossed disparity processing. Since much of the previous research has documented the asymmetry with RDSs using temporal duration thresholds as the dependent measure, the present study detected it with local targets using RT and accuracy as the dependent measures. Also in support of previous research, the present study detected a high incidence of "anomalous" behavior in stereopsis. Because of this high incidence and the fact that it may decrease with practice, we feel that the term "stereoanomalous" should be reserved for describing behavior, and not for describing observers per se. Although these results are consistent with a scheme of disparity detector pooling, other research supporting a scheme of continuous disparity tuning is too convincing to be ignored. Therefore, we have interpreted results in support of the latter scheme, arguing that a multicomponent, or perhaps sequential, process of disparity processing can provide results supporting both competing theories.

\section{REFERENCES}

ADAM, J. J., \& VAN VEGGEL, L. M. A. (1991). Discrete finger response latencies in a simple reaction time task. Perceptual \& Motor Skills, 73, 863-866.

ANDERSEN, G. J. (1990). Focused attention in three-dimensional space. Perception \& Psychophysics, 47, 112-120.

BaDCoCK, D. R., \& SchoR, C. M. (1985). Depth-increment detection function for individual spatial channels. Journal of the Optical Society of America A, 2, 1211-1216.

BreITMEYER, B., JULeSz, B., \& KROPFL, W. (1975). Dynamic randomdot stereograms reveal up-down anisotropy and left-right isotropy between cortical hemifields. Science, 187, 269-270.

Cormack, L. K., Stevenson, S. B., \& Schor, C. M. (1993). Disparity- tuned channels of the human visual system. Visual Neuroscience, $\mathbf{1 0}$, 585-596.

Downing, C. J., \& Pinker, S. (1985). The spatial structure of visual attention. In M. I. Posner \& O. S. M. Martin (Eds), Attention and performance $X I$ (pp. 171-187). Hillsdale, NJ: Erlbaum.

FolEY, J. M., \& RICHARDS, W. (1974). Improvement in stereoanomaly with practice. American Journal of Ophthalmology \& Physiological Optics, 51, 935-938.

Heister, G., Ehrenstein, W. H., \& Schroeder-Heister, P. (1986). Spatial S-R compatibility effects with unimanual two-finger choice reactions for prone and supine hand positions. Perception \& Psychophysics, 40, 271-278.

JuLESZ, B. (1971). Foundations of cyclopean perception. Chicago: University of Chicago Press.

JULESZ, B., BREITMEYER, B., \& KROPFL, W. (1976). Binocular-disparitydependent upper-lower hemifield anisotropy and left-right hemifield isotropy as revealed by dynamic random-dot stereograms. Perception, 5, 129-141.

Lachnit, H., \& Pieper, W. (1990). Speed and accuracy effects of fingers and dexterity in 5-choice reaction tasks. Ergonomics, 33, $1443-1454$

LeGGE, G. E., \& Gu, Y. (1989). Stereopsis and contrast. Vision Research, 29, 989-1004.

LEHKY, S. R., \& SEJNOWSKI, T. J. (1990). Neural model of stereoacuity based on a distributed representation of stereo disparity. Journal of Neuroscience, 10, 2281-2299.

LEVAY, S., \& VoIGHT, T. (1989). Ocular dominance and disparity coding in cat visual cortex. Visual Neuroscience, 1, 395-414.

Manning, M. L., Finlay, D. C., Neill, R. A., \& Frost, B. G. (1987). Detection threshold differences to crossed and uncrossed disparities. Vision Research, 27, 1683-1686.

McKee, S. P., Welch, L., TAYlor, D. G., \& Bowne, S. F. (1990). Finding the common bond: Stereoacuity and the other hyperacuities. Vision Research, 30, 879-891.

Newhouse, M., \& UTTAL, W. R. (1982). Distribution of stereoanomalies in the general population. Bulletin of the Psychonomic Society, 20, 48-50.

O'Shea, R. P., \& BlaKe, R. (1987). Depth without disparity in randomdot stereograms. Perception \& Psychophysics, 42, 205-214.

Patterson, R., Cayko, R., Short, G. L., Flanagan, R., Moe, L., TayLOR, E., \& DAY, P. (1995). Temporal integration differences between crossed and uncrossed stereoscopic mechanisms. Perception \& Psychophysics, 57, 891-897.

Patterson, R., \& Fox, R. (1984). The effect of testing method on stereoanomaly. Vision Research, 24, 403-408.

PogGio, G. F., \& Fischer, B. (1977). Binocular interaction and depth sensitivity in striate and prestriate cortex of behaving rhesus monkey. Journal of Neurophysiology, 40, 1392-1405.

Poggio, G. F., GonZalez, F., \& Krause, F. (1988). Stereoscopic mechanisms in monkey visual cortex: Binocular correlation and disparity selectivity. Journal of Neuroscience, 8, 4531-4550.

Previc, F. H., \& Blume, J. L. (1993). Visual search asymmetries in three-dimensional space. Vision Research, 33, 2697-2704

Previc, F. H., Breitmeyer, B. G., \& Weinstein, L. F. (1995). Discriminability of random-dot stereograms in three-dimensional space. International Journal of Neuroscience, 80, 247-253.

Previc, F. H., Weinstein, L. F., \& Breitmeyer, B. G. (1992). Visual attention and perception in three-dimensional space (Tech. Rep. No. AL-TR-1991-0119). Brooks Air Force Base, TX: Armstrong Laboratory.

RICHARDS, W. (1970). Stereopsis and stereoblindness. Experimental Brain Research, 10, 380-388.

RICHARDS, W. (1971). Anomalous stereoscopic depth perception. Journal of the Optical Society of America, 61, 410-414.

SNODgRass, J. G. (1975). Psychophysics. In B. Scharf \& G. S. Reynolds (Eds.), Experimental sensory psychology (pp. 17-67). Glenview, 1L: Scott, Foresman.

Stevenson, S. B., Cormack, L. K., Schor, C. M., \& Tyler, C. W. (1992). Disparity-tuned mechanisms of human stereopsis. Vision Research, 32, 1685-1689. 
YANG, Y., \& BLAKE, R. (1995). On the accuracy of surface reconstruction from disparity interpolation. Vision Research, 35, 949-960.

\section{NOTES}

1. The index finger, lying on the "l" key ("front" response) was positioned closer to the observer than the middle finger, lying on the " 2 " key ("back" response). Evidence from Lachnit and Pieper (1990), Adam and Van Veggel (1991), and Heister et al. (1986) indicates that there are no intrinsic speed differences between these two fingers.

2. O'Shea and Blake (1987) have shown that observers perceive depth from uncorrelated regions in RDSs in the absence of retinal disparity, and that the sign of such "rival depth" can be predicted from fixation disparity. It is conceivable that the reversal we report is related to rival depth. However, this requires some speculation, since diplopia in local stereograms is not completely analogous to decorrelation in RDSs. Decorrelation inhibits veridical depth perception by reducing disparity information. Diplopia does not necessarily have this effect. As seen in Figures 2-9, our diplopic stimuli were almost always perceived at least as accurately as fused stimuli. This suggests that these targets are under the upper depth limit, and therefore, contain adequate disparity information.

3. Using various methodologies, several studies have produced conflicting findings regarding the direction from fixation attention favors. For example, Andersen (1990) suggested that it favors the uncrossed direction, Downing and Pinker (1985) suggested that it favors the crossed direction, and Previc and Blume (1993) suggested that it favors neither direction. Previc, Weinstein, and Breitmeyer (1992) addressed the topic but were unable to reach a conclusion, citing the difficulty with measuring attention in 3-D space.

4. D.D.L. experienced the reversal during the collection of pilot data. Unable to see uncrossed stimuli as distal, he practiced with some uncrossed stimuli, displayed indefinitely. The percept was always a proximal one, until on one trial, the target suddenly receded continuously to its proper distal position. Thereafter, he continued to see them correctly.

(Manuscript received October 23, 1995; revision accepted for publication April 4, 1996.) 